\title{
A DIGITAL LEGO-BASED LEARNING ENVIRONMENT FOR FRACTION ORDERING
}

\author{
0. C. Agbonifo ${ }^{1, *}$ and A. O. Ogunmoroti ${ }^{2}$ \\ 1, 2 Department of Computer Science, Federal University of TeChnology, AKURe, ONdo State, NiGERIA \\ E-mail addresses:1 ocagbonifo@futa.edu.ng, ${ }^{2}$ ogunmorotiabiodun@gmail.com
}

\begin{abstract}
Learning environment is a process where learning is activated or enabled and the resources to support it are made available to a learner in order to construct and acquire knowledge. An environment must be simulated with features that enable learning to be fascinating, motivating and required mental process for critical thinking. What is to be learnt is crucial in learning and the focus of learning as a domain knowledge is fraction ordering, which is one of the topics in Mathematics. Hence, this paper develops a digital Lego-based learning environment for fraction ordering.
\end{abstract}

Keywords: Learning environment, digital Lego-based, fraction ordering, educational technology

\section{INTRODUCTION}

Many educationists from different perspectives have defined educational technology but all tends towards achieving the same objectives. The application of technology in learning has brought about innovations into educational system where the roles of students and learners have tremendously transformed in a virtual learning community than in a traditional or conventional learning community. With the assistance of the new technology, teaching and learning have taken the form of creative act and application of game technique to allow students use high order of cognitive or mental activities that usually transform learning to acquisition of knowledge. Different researchers within the principles of learning theories have defined learning. Learning can be perceived within the contexts of discuss but all tends to achieve a unique key of acquiring knowledge to promote national development.

Learning environment is described as varied geographical locations, contexts, and cultures in which students learn to acquire skills. It has been a great challenge on the part of teachers to create interesting environments that encourage active and constructive participation of students. Learning environment has both direct and indirect influence on student learning outcome. The context in which learning takes place, as well as the tools' mediation play a crucial part providing opportunities for active, exploratory and personally significant learning for the individual [13]. Learning involving game engagement explores creativity and critical thinking that make use of cognitive activities promoting active participation in learning process. Many researchers have explored this new wave of research and the findings have been so promising. The use of game environment has been proved as added advantage to students' learning because of hands-on practical experience. The construction of Lego-based learning will bring a great attraction to students to actively learn and promote their reasoning faculty to broaden their knowledge on the area of subject matter.

Mathematics is described in terms of content such as number, space and measurement; procedures for computing, constructing or applications across a wide range of situations [5]. Booker [5] further described it as a way of thinking, which allows concepts, processes and their uses to be built up, problems to be explored and solved, assumptions to be made and examined, and complex ideas to be communicated in precise and concise ways. Having an ability to think through with mathematics has replaced the way students memorise set procedures and now focus of mathematics at all levels of learning is the step-by-step solution to routine problems. 
Anthony and Wilshaw [4] stressed that quantities in terms of "how many" and "how much" can be use to describe fractional numbers and is very important in everyday-life situations. From the literature, fraction did not originally have a mathematical sense, it was formed from a Latin word which means "to break" [7\}. Formal definition in Algebra is: $q(x)=h(x) / g(x)$ where $\mathrm{h}(\mathrm{x})$ and $\mathrm{g}(\mathrm{x})$ are rational numbers and $\mathrm{g}(\mathrm{x})$ cannot be equal to zero. Fraction is a ratio of two expressions or numbers other than zero and it is also a part of a whole [14].

Yoshida and Shinmachi [15] stressed a notion that fractions, ratios, and proportions are described as the most mathematically rich and cognitively complex areas in primary school mathematics, which are difficult for teachers to teach and for students to learn. Mousley and Kruz [16] proposed some standards for learning fractions, which include developing an understanding of fractions using manipulative, visual models, and illustration to name and represent unit fractions as part of a whole or a set of objects, understanding and recognizing the meaning of numerator and denominator in the symbolic form of a fraction. Hence, there is need to address this difficulty in learning fraction for students to get acquainted with fractions, this research paper focuses on using a digital Lego-based learning environment to help the pupils in comparing fractions with like and unlike denominators to reduce the difficulty encountered by pupils when working with fractions. It makes use of a game environment to make learning attractive that will lead to successive learning outcome.

\section{OVERVIEW OF COGNITIVE AND CONSTRUCTIVE LEARNING THEORIES}

Cognitive and developmental psychologists viewed learning as a dynamic process where learners construct their own knowledge by interacting with the world, while constructivism is a process where teachers and educational technology do not impose steps, procedure and rigid structure on students but rather they act as the architecture for learning environments that makes easier for students to build their own knowledge [11]. Cognitive development is described as the mental process by which knowledge is acquired, stored, and retrieved to solve similar problems. There are number of theories that relate to cognitive and constructive approach to learning where some are concerned with cognitive development, others are concerned with social aspects, and still others are concerned with the role of context [11].
Gillian and O'Guinn [11] reported that Piaget's cognitive developmental theory argued that children are to reconstruct their understanding of any phenomenon through active reflections on objects and events until adult perspective is achieved. Piaget's theory emphasized that children move through four developmental stages from infancy to adolescence. In this theory, schema is used to represent a mental structure that adapts to environmental pattern and the internal mental processes that are responsible for the restructuring of the child's schemata are assimilation and accommodation. Integrating a new information with existing knowledge as a cognitive process called assimilation while restructuring of prior knowledge is to make a new experience fit in the newly formed schema and the change that occurs in the mental structure of the child is referred to as accommodation. The mostly two areas that the influence of Piaget's theory has on instructional design, teaching models, and educational technology are discussed as follows:

a. Inquiry Training

Flavell [9] provided a detailed discussion of three operations, which are combinational reasoning, propositional reasoning, and hypothetical-deductive reasoning that adolescents gradually acquire during the formal training of their development. Combinational reasoning is described as the ability of the adolescent to consider several different factors at the same time to solve problems. This reasoning power provides the child with the ability to look at problems from an integrated approach. Propositional reasoning is the characteristics that young adolescents acquire to reason on the basis of assumption and proposition to solve problems. This ability extends to abstract thinking that is acquired during the formal stage. Hypothetical-deductive reasoning allows the young adolescent to consider different hypotheses in dealing with problem. The focus of inquiry training is to assist students develop a sense of the independent inquiry method mostly in a disciplined way.

b. Discovery learning

Gilliani and O'Guinn [11] reported that the work of Bruner and Papert were inspired by Piaget's theory of learning and developed the discovery learning method. Discovery learning focuses on exploring, experimenting, doing research, asking questions, and seeking answers. It also assist learners to take control of their own learning, use of high order of mental thinking, and helps students recall relevant facts. 


\section{RELATED WORKS}

Resnick et al [12] described the development of the Lego/Logo environment and discussed the role of design and invention activities in the classroom, drawing on the experiences of the elementary-school children. The researchers tried to situate the Lego/Logo materials in a broader learning environment and making it an approach for learning and thinking. Furthermore, many ways were examined by the researcher to concretise the concealed abstract ideas on why Lego/logo environment fits in the general trend towards "handson" education. Also, reasons that brought about the feeling that the Lego/Logo environment goes beyond traditional hands-on activities were stated, which were because the environment makes use of familiar materials and real activities, and that the environment offers multiple paths of learning. Nevertheless, the researchers failed to investigate the appropriate roles of teachers in open-minded design activities like LEGO/Logo, and the type of skills that teachers needs to meet the roles of differences among students and lots more.

Lee and Shanks [10] developed a digital game for the instruction of fractions where a basic fraction game model was designed to focus on the topic of comparing fractions sizes with the learning objective of ordering fractions. The researchers work was inspired by Amory [2]. The digital game environment uses several game resources such as game objects, sprites, events, actions and rooms. The game includes features that realised the instructional factors and students' needs stressed in the game model. The game environment was structured in line with the instructional considerations such as conceptual analysis of fractions, students' misconceptions, instructional strategies, students' strategies, and the relationship of the game to the New Zealand Number framework. The main objective of the fraction game is to order fractions through a story of forming staircases to go up to a tower. There were three acts designed for the game. The focus of Act 1 is to visualise representations of fractions while that of Act 2 is to make sense of fraction symbols. The Act 3 is to partition fractions for the given denominators and to label the fractions. All these were implemented through the concrete interfaces of the Visualisation space (story line), Elements space (graphics, sound and technology), Actor's space (interaction and gestures) and Problem space (visual, logic, mathematical, short-term memory and manipulation) to express the abstract interfaces (critical thinking, discovery, goal formation, goal completion and practice) in the visualisation space, Elements space (fun) and Actor's space (drama). The result was not presented in the research work to show the suitability of the system to underlined objectives.

Glezou [13] focused on the development of learning environments with the use of Logo programming language in didactic praxis to promote exploratory and collaborative learning in primary and secondary education and teacher training. In the research work, the special features of online learning environment were discussed, which involves creating new conditions for learning and demonstrating a variety of new possibilities for alternative forms of communication, interaction and cooperation by supporting collaborative learning. The research work explored the use of pre-constructed micro worlds for the construction of new artifacts with a gradually increasing degree of complexity which encourages the systematisation of knowledge and bridges the gap between the simple and the more complex.

Kruse [1] described learning as a process whereby learners make meaning of new ideas through connection to their current conceptual framework. Well-established learning theories are grouped into two basic categories such as behavioural learning theories and cognitive learning theories. The behavioural learning theory (BLT) treats the mind as a black box and focuses on changes in observable behaviours. BLT is attractive because of its simplicity, ability to explain phenomena, clear implications for pedagogical practice, and basis in controlled research [8]. Cognitive learning theories emphasis the role of active mental processing, that is, it focuses on how students makes meaning of new information and experience. Cognitive learning theories include constructive learning theory (CLT), development learning theory (DLT) and Social learning theory (SLT) which emphasise different factors that affect the meaning-making process.

In Cho et al [3], the study provided the participants with a clear objective, which is creating a patternmaking box for manipulation and construction of activities to achieve the objective. The objective corresponds to pattern generation, which naturally induces the concept and proper understanding of symbols in the design. With this design, the researchers tried to find out if the environment provided for exploring pattern generation of symbols was appropriate and through the analysis and interpretation of participants' responses to the questionnaire, it was observed that some students 
constructed the general expression that produces patterns, using '(n)' variable perfectly while some of the students that could not figure out the meaning of variables with their description in the next task were provided with more elaborate design through followup studies.

Cho et al [6] developed a logo-based mathematics created by you (MCY) learning environment for the polycube pattern activities in the elementary school mathematics textbook in Korea. The learning environment attracts the deployment of Web 2.0 using Java Mal micro world for students to interactively create and explore pattern objects; with available resources such as virtual manipulative and expressive tools to support students' thought process for pattern generalisation. With the interaction of the students with pre-test and post-test tasks, the researchers analysed students' responses to determine students' algebraic thinking based on their symbolic pattern expressions. The results obtained showed that students' pattern reasoning became more structured and sophisticated, and the designed learning environment was suitable to support students' algebraic thinking for pattern generalisation in the context of pattern manipulation and construction.

\section{SYSTEM DESIGN}

This section presents the architecture, flowchart, and the design process of the system. This research work was motivated by the work of Lee and Shanks [10] and would be suitable to complement the teaching and learning of fraction in the traditional classroom setting of the primary school education in Nigeria. The game also has a background story that has link with the underlined objective of learning fraction ordering. A boy has to get home before a given time elapsed and at the same time to pluck some fruits before entering the house. To pluck the fruit, the boy needs to break some fraction cube in ascending order. The story provides a meaningful context for the player to apply the knowledge of fractions where the learning objective is to break the fraction cube in ascending order. Hence, the topic of ordering fractions as one of the critical aspects of fractions is focused. The rule of the game is to break the fraction cubes in ascending order and then, pluck the available fruits, but any attempt to violate the rule of the game will attract a penalty of reduction in the time given. The goal is to get home before the given time elapsed. If the fraction cubes are broken in the correct order, the score is increased and the player will have the opportunity of plucking the fruits. The outcome of the game is to resolve the conflict in the limited understanding of the mental picture of fractions and to compare different sizes of fraction cubes. Positive feedback and rewards are given when there are misconceptions, conflict and understanding to make the game so attractive. Digital timing synchronisation makes the game interesting. Fraction cubes stand like obstacles blocking the player from plucking the fruit and this makes the game tasking but interesting.

\subsection{System Architecture}

The architecture is depicted in Figure 1 which consists of the following components such as game input, message handling, game logic applied on game processing unit; and display game output in form of graphics, sound and text. The game input is the process of entering data into digital Lego-based learning system for fractional ordering. Message handling is an interface providing events to be communicated within entities to make them perform their usual activity (participating in the game). The message also contained additional information such as a sender address or information, $a$ recipient address or information and a time stamp. The game logic uses the AND and OR operator and function where the game player chooses the valid action to perform based on the event or chains of events that occurred in the game. In AND type, all events must be satisfied and then the attached action can be performed while in OR type, one of the events must be satisfied and then the action can be performed. Game output is the resultant effect of the processing of the input supplied by the game player to play according to the rule of the fractional ordering game.

\subsection{System Flowchart}

The flowchart of the digital Lego-Based Learning Environment for fractional ordering is depicted in Figure 2, which demonstrates the flow of the events and actions that are taking place in the game. At the start, the system reduces the given time at 120 seconds by one per each tick of clock. The game continue running as far as the given time is greater than zero (0), waiting for input from the user or player. The means of input is through mouse and keyboard, the keyboard is to move the player from one location to another while the mouse is used to break the fraction cube by clicking it one at a time. If the keyboard is used as the means of input then the player can move towards any of the four directions (right, left, front or back) but if the input is from the mouse, the game makes a decision based on the 
fraction cube that was clicked; if the fraction cube is clicked in ascending order among the list then each fraction cube is broken and the player score is increased by 50 otherwise the given time is reduced by 5 . The game ends when the given time has been exhausted or when the user plays within time. The game can be play repeatedly as the player desires and this is to assist the player to acquire the skill of the game's objective.

\subsection{Design Process}

The application-programming interface known as Construct 2 is used to develop the system in stepwise process. The design process for constructing the game environment is shown in Figure 3 which comprises of various objects: an image of a person playing the game which has the ability to move from one location to another, bricks in Lego shape that was used to form different shapes to restrict the movement of the player, fraction cubes for the player to break that lead to fruits for the player to pluck and increase his score. Likewise, a heads-up display (HUD) was added to display the given time and the score. The Figure 4 shows the interface to display the score of the user when the given time has been exhausted.

Figure 5 shows the game at the running stage. The game is running on a browser and the given time continues decreasing by one per each clock tick. The actions of the user will either increment the score of the player if played according to the rule of the game or decrement the time and the score of the player if played against the rule. The time constraint in the game environment makes it competitive and interesting to the user.

When the player collides with any fruit, the fruit object is destroyed but unless the user breaks the fraction cubes standing as barricade, the player would not be able to pluck the fruit. For the player to increase the score, identification of the fraction numbers by arranging in ascending order is very important because this is the act of learning. When the game was not played according to the rule, this will have effect on the overall learning performance. The game automatically ends when the given time is exhausted.

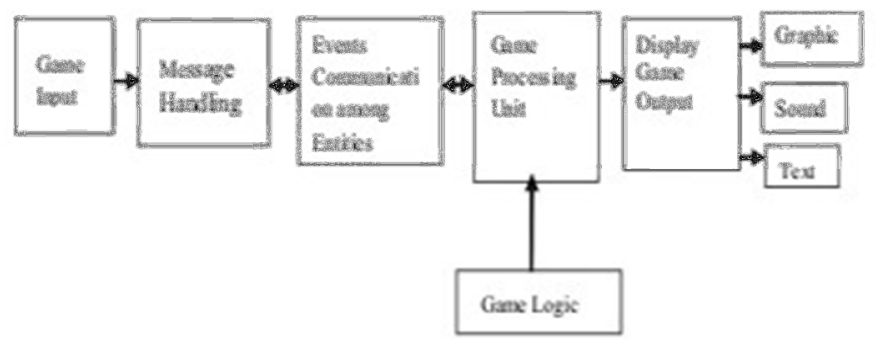

Figure 1: Architecture of Digital Lego-Based Learning Environment

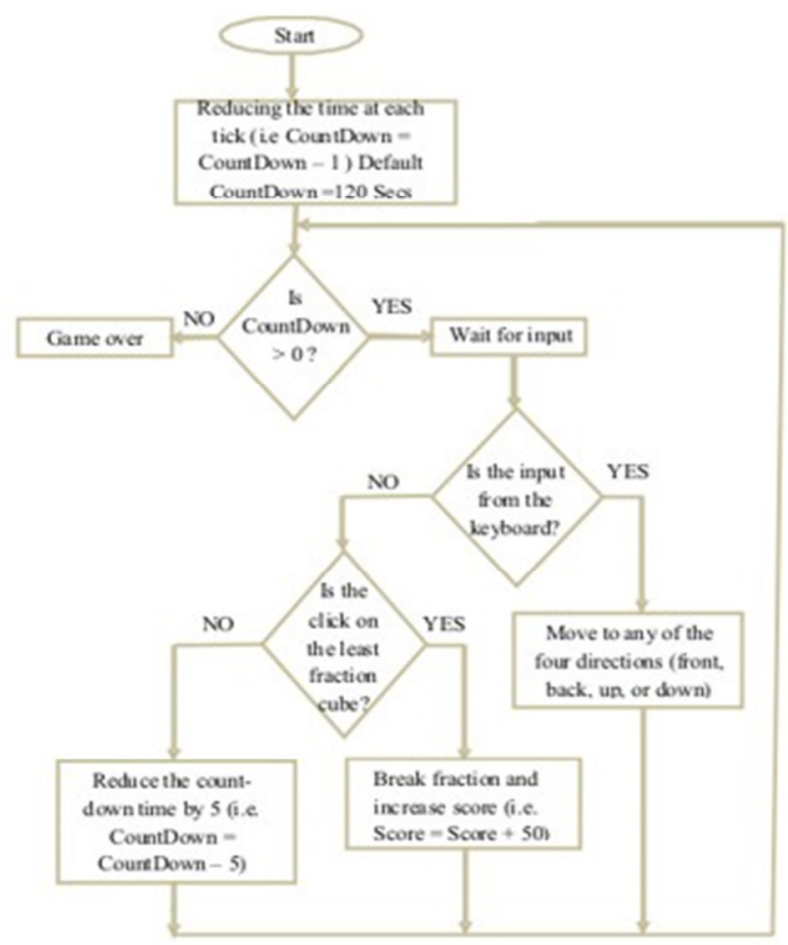

Figure 2: Flowchart of Digital Lego Based Learning Environment

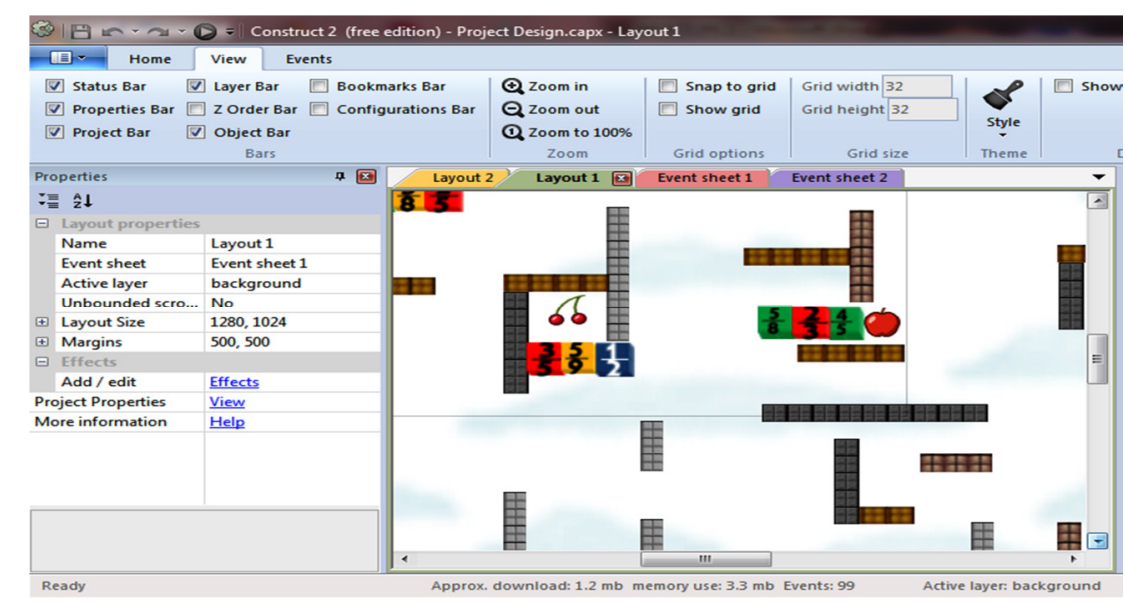

Figure 3: Design Process for Constructing Digital Lego-Based Learning Environment 


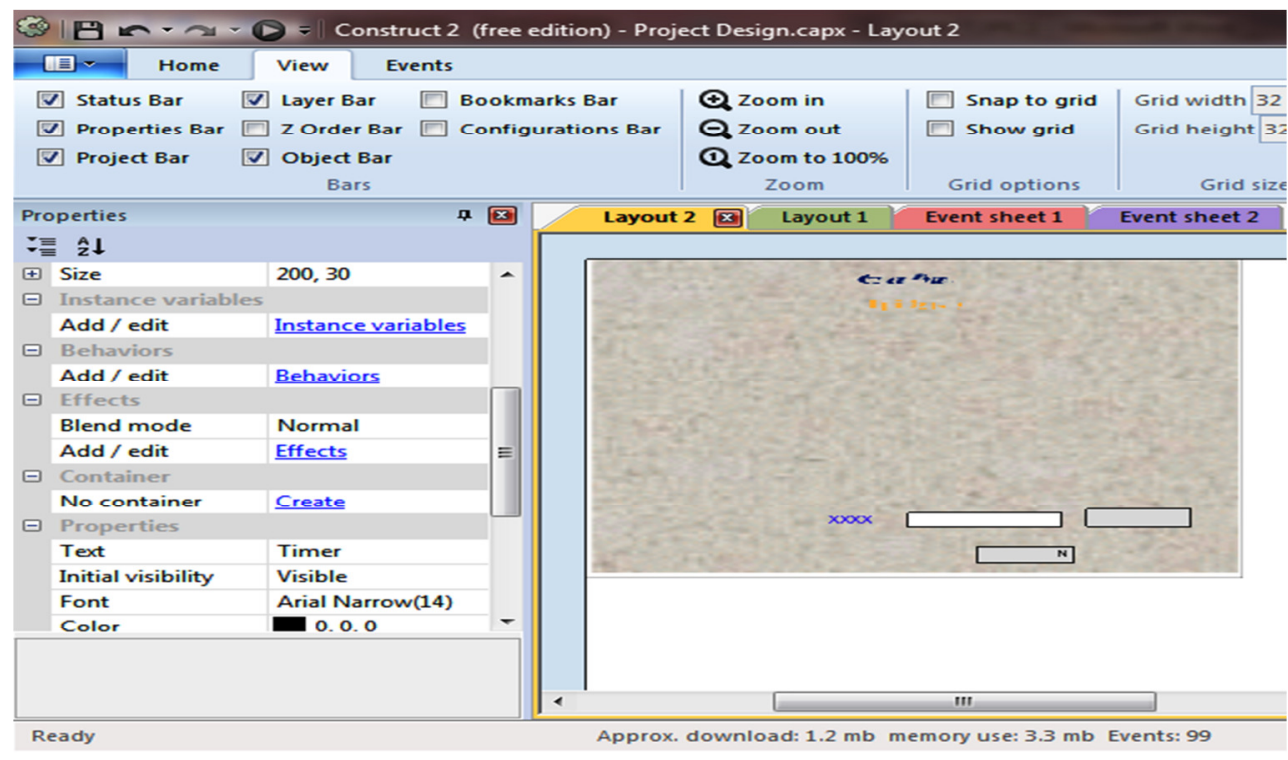

Figure 4: Design Process to Display Score

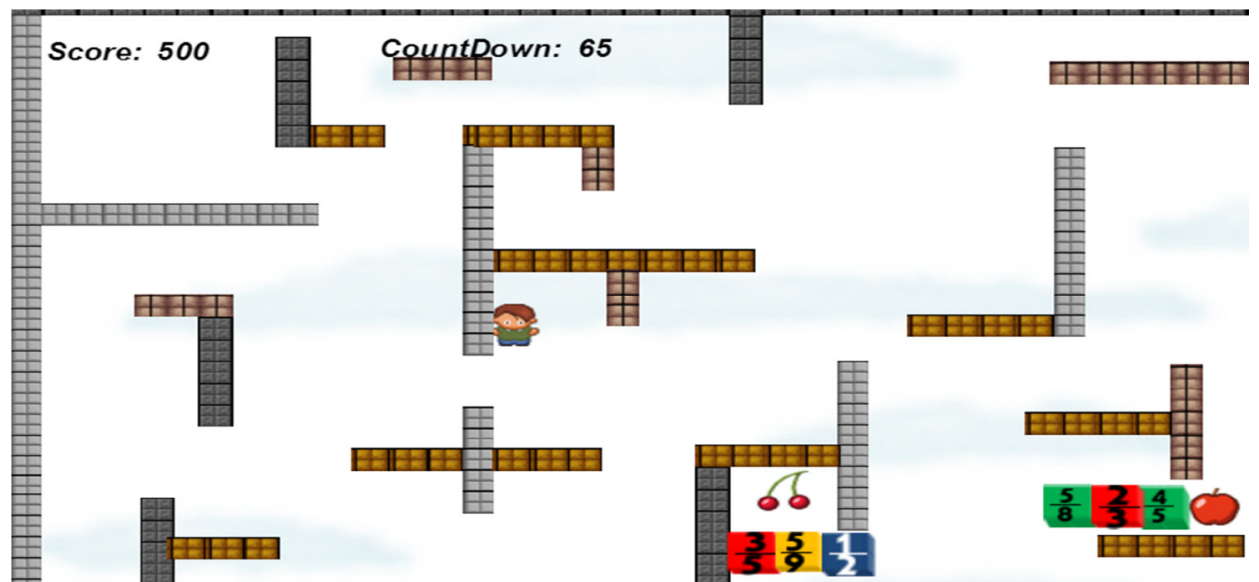

Figure 5: Digital Lego-Based Learning Environment at Run Time

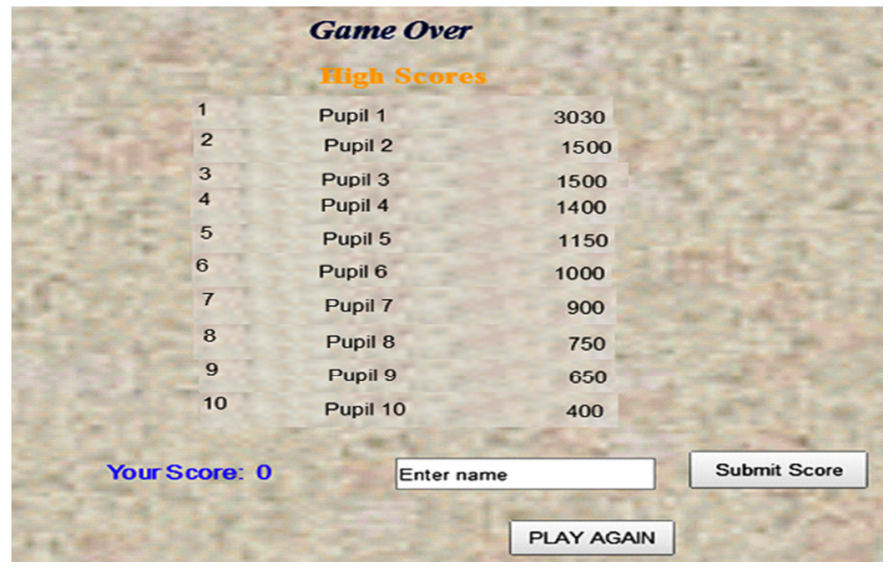

Figure 6: Game output

\section{DISCUSSION AND RESULT}

The system was tested by subjecting it to a set of ten pupils of primary four in one of the private primary schools situated around the Federal University of Technology, Akure. These set of pupils were gainfully engaged in the game process to learn the act of fraction ordering and to demonstrate their abilities. The outcome of the game played by these pupils is displayed in Figure 6, which is arranged according to their scores.

\section{CONCLUSION}

Mathematics is a subject that deals with numbers and invariably requires the act of thinking to inductively or deductively connect to meaning concepts or ideas. Gaming is also an act of hands-on process to make the learner carry out the aspect of doing, thinking out and self-independence in learning process with little or no supervision or support. The system was developed to make the understanding about "fraction ordering" much simplified to learn as a topic in mathematics. It makes learning environment attractive, interactive and engages with cognitive and constructive process. It will assist to build mental skills of pupils. The system was tested and the result obtained 
demonstrates that the system is suitable for the underlined objective.

\section{REFERENCES}

[1] Kruse, J. "Learning Theories: Pillars of Teacher Decision-Making", IOWA Science Teacher Journal, $36(2)$, pp. $1-6,2009$.

[2] Amory, A. "Game object model version II: A Theoretical Framework for Educational Game Development", Educational Technology, Research and Development, 55(1), pp. 51-77, 2007.

[3] Cho, H., Kim H., Shin, D. and Lee, J. "Exploring Pattern Generalization in the Logo-based Microworld", Proceedings of the Seventeenth Asian Technology Conference in Mathematics, December 16-20, Bangkok, Thailand, pp. 1 - 10, 2012.

[4] Anthony, G. and Walshaw, M. "Effective Pedagogy in Mathematics/Pangarau: Best Evidence Synthesis Interaction", Ministry of Education, Wellington, New Zealand, 2007.

[5] Booker, G. "Thinking Mathematically - Making Sense and Solving Problems", The Mathematics Education into $21^{\text {st }}$ Century Project, Universiti Technologi Malaysia, November 25-December 1, pp.49-53, 2005.

[6] Cho, H. H., Lee, J. Y. and Song, M. H. “Design of a Logo-Based Learning Environment for Pattern Generalization", Proceedings of World Conference on Education Media, Hypermedia and Telecommunications, Columbia, June 24-28, pp. 807-813, 2013.

[7] Farlex, "Definition of Fraction", www.thefreedictionary.com/fraction, Accessed on August 24, 2014.
[8] Collins, A. "How Students Learn and How Teachers Teach", Bypee, R. W. (Ed.), Learning Science and the Science of learning, National Science Teachers Association Press, Arlington, VA, 2002.

[9] Flavell, J. H. "The Development Psychology of Jean Piaget", Stanford University Press, 1963.

[10] Lee, Y. L. and Shanks J. "Developing a Digital Game for the instruction of Fractions", Proceedings of the Eleventh International Congress on Mathematical Education, Mexico, July 7-12, pp. 1 - 8, 2008.

[11] Gilliani, B. B. and O'Guinn, C. “Cognitive Theories and the Design of E-learning environments", Global Health Informatics Education, Vol. 109, pp.143-151, 2004.

[12] Resnick, M., Ocko, S. and Papert, S. "LEGO, Logo, and Design", Children's Environments Quarterly, 5 (4), pp. 14-18, 1988.

[13] Glezou, K. "Development of Learning Environments with use of Logo Programming Language in Teaching Praxis", PhD. dissertation submitted to the Department of Informatics and Telecommunications, National and Kapodistrian University of Athens,2010.

[14] Smith, S. "Maths is Fun", www.mathisfun.com/ fraction, Accessed on August 23, 2014.

[15] Yoshida, H. and Shinmachi, Y. "The Influence of Instructional Intervention on Children's Understanding of Fractions", Japanese Psychological Research, 41(4), pp.218-228,1999.

[16] Mousley, K. and Kruz, C. "Difficulty of Fraction Learning; Dilemma in Mathematical Teaching", SWCED Houston, TX, 2012. 